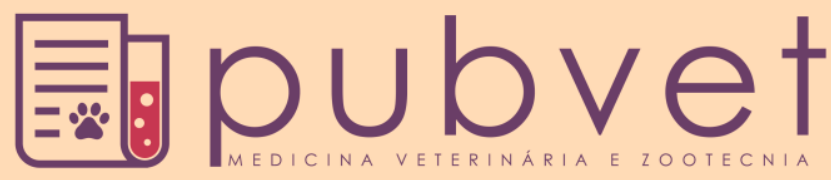

https://doi.org/10.22256/pubvet.v12n6a113.1-4

\title{
Técnica de fixação segmentar modificada com pinos de Steinmann em fratura Lombosacral entre L7 e S1: Relato de Caso
}

\author{
Samuel Otemi de Sousa Silva ${ }^{1^{*}}$, Bruno Martins Araújo ${ }^{2}$, Duan Kessiley Torres de \\ Sousa $^{1}$, Daniele Benigno Mota ${ }^{1}$, Francisco Lisboa Sousa Junior ${ }^{3}$, Ramon Matheus \\ Lima $\operatorname{Matos}^{10}$
}

${ }^{I}$ Graduando em Medicina Veterinária da Universidade Federal do Piauí.

${ }^{2}$ Médico Veterinário Cirurgião do Hospital Veterinário Universitário da Universidade Federal do Piauí.

${ }^{3}$ Residente em Clínica Médica e Cirúrgica do Hospital Veterinário Universitário da Universidade Federal do Piauí;

*Autor para correspondência. E-mail: samuelotemi@gmail.com

\begin{abstract}
RESUMO. A síndrome da cauda equina acontece pelo estreitamento do canal causando isquemia das raízes nervosas L6, L7, S1 e S2 origem do nervo pudendo e ciático. O objetivo deste trabalho é relatar um caso de uma cadela, sem raça definida de aproximadamente 9 meses de idade, atendida no hospital veterinário do Piauí, diagnosticada com fratura Lombossacral entre 17 e S1, tratada cirurgicamente por meio da técnica de Fixação segmentar modificada com pinos de Steinmann. A técnica se mostrou efetiva na estabilização e imobilização da fratura, mostrando uma boa resposta na recuperação do paciente.
\end{abstract}

Palavras-chaves: cauda equina, trauma, fixação segmentar

\section{Fixation technique segmental modified with Steinmann pins in a Lumbosacral fracture between L7 and S1: Case Report}

ABSTRACT. Equine tail syndrome occurs due to narrowing of the canal causing ischemia of the nerve roots L6, L7, S1 and S2 origin of the pudendal and sciatic nerve.The objective of this work is to report a case of a bitch, with no defined breed of approximately 9 months of age, attended at the veterinary hospital of Piauí, diagnosed with a lumbosacral fracture between 17 and S1, surgically treated by the segmental fixation technique modified with pins of Steinmann. The technique was effective in the stabilization and immobilization of the fracture, showing a good response in the recovery of the patient.

Keywords: equine tail, fracture, segmental fixation

\section{Técnica de fijación segmentada modificada con pinos de Steinmann en fractura Lumbosacra entre L7 y S1: Reporte de un caso}

RESUMEN. El síndrome de la cola equina ocurre por el estrechamiento del canal causando isquemia de las raíces nerviosas L6, L7, S1 y S2 origen del nervio pudiendo y ciático. El objetivo de este trabajo es relatar un caso de una perra, sin raza definida de aproximadamente 9 meses de edad, atendida en el hospital veterinario de Piauí, diagnosticada con fractura Lombosacral entre 17 y S1, tratada quirúrgicamente por medio de la técnica de Fijación segmentada modificada con pines de Steinmann. La técnica se mostró efectiva en la estabilización e inmovilización de la fractura, mostrando una buena respuesta en la recuperación del paciente.

Palabras claves: rabo equino, trauma, fijación segmentada 


\section{Introdução}

As lesões lombo sacrais causam estreitamento do canal vertebral e compressão das raízes nervosas, causando a síndrome da cauda equina. (Prata, 1998).

O diagnóstico inicia-se com o exame físico geral pela palpação do examinador na região lombo sacral, sem causar dor. Deve-se aplicar certa pressão as vertebras torácicas e lombares a fim de avaliar o nível de dor e a extensão da lesão e presença de lesão no neurônio motor inferior. Alfinetadas em dermatómos específicos ajudam a perceber a localização das raízes nervosas irritadas (Feitosa, 2014).

A compressão das raízes nervosas no segmento da primeira vertebra sacral (S1) a terceira vertebra sacral (S3) da origem ao nervo pudendo. A sua compressão tem como consequência a perda do reflexo de micção e de defecação, perda do reflexo perianal, retenção fecal, a cauda se torna irresponsiva. Como consequência há o acúmulo de urina na vesícula urinária que se torna distendida o paciente passa a urinar na forma de gotas pelo esfíncter relaxado. O esfíncter anal se dilata e as fezes saem pelo reto de maneira involuntária. Lesões acima de S1 e S3 o reflexos do esfíncter anal vão estar presentes (Chrisman, 1985; Prata, 1998).

Os sinais clínicos da síndrome da cauda equina incluem: Dor nas costas, flexão do dorso, paresia, atrofia muscular na distribuição do nervo isquiático, debilidade da cauda, distúrbios nos esfíncteres levando a incontinência urinaria e fecal. A síndrome se não tratada evolui para paralisia dos membros pélvicos. (Inzana \& Wood, 2008; Prata, 1998).

No exame neurológico, avalia-se o posicionamento proprioceptivo, o movimento e apoio, reflexos dos membros, reflexo perineal, avaliação da dor superficial e profunda. Outros sinais, incluem angulação aumentada da pelve com a coluna indicando luxação ou trauma. A radiografia simples é indicada quando se suspeita de doença focal ou multifocal na coluna vertebral. (Chrisman, 1985).

As fraturas na coluna vertebral e principalmente as fraturas no segmento da cauda equina são comuns na clínica de pequenos animais. O presente relato visa contribuir para o estudo e o emprego das técnicas de correções nas fraturas lombo sacrais.

\section{Relato de Caso}

Uma cadela, sem raça definida, com 9 meses de idade, com massa corporal de $6,15 \mathrm{~kg}$, foi atendida no hospital veterinário, possuindo histórico de atropelamento, há dois dias. Apresentava fezes enegrecidas, estava urinando normalmente, com mucosa normocorada e linfonodos não reativos. No exame físico o paciente apresentava com sensibilidade abdominal e crepitação na região sacral, muita dor na região lombar e sacral, angulação do sacro e coluna aumentada.

No exame neurológico, se apresentava com dor superficial ausente nos membros pélvicos e dor profunda presente, com reflexo patelar aumentado, com ausência de reflexo perineal, paresia dos membros pélvicos, com propriocepção diminuída. Foram solicitados os seguintes exames: ultrassonografia e radiografia da pelve e do sacro.

$\mathrm{Na}$ radiografia observou-se a fratura na coluna vertebral entre as vertebras 17 e S1 (Figura 1. A). $\mathrm{O}$ paciente foi encaminhado para cirurgia cuja técnica utilizada foi à técnica de fixação espinhal segmentar modificada com uso de pinos de Steinmann. Durante o pré-operatório foi prescrito Carprofeno, 4,4 mg/ kg, BID por via oral. Para o pós-operatório foi prescrito Carprofeno, $4,4 \mathrm{mg} /$ $\mathrm{kg}$, BID por via oral por 3 dias, e Tramadol $4 \mathrm{mg} / \mathrm{kg}$ a cada 8 horas, enrofloxacina $4 \mathrm{mg} / \mathrm{kg}$ $\mathrm{BID} / \mathrm{IV}$ e cefalexina $30 \mathrm{mg} / \mathrm{kg}$ BID por 24 horas.

Segundo Curtis (2014), a técnica de fixação espinhal segmentar modificada com uso de pinos de Steimann caracteriza-se por introduzir o pino de Steinmann através do aspecto lateral da asa do íleo entre espaço entre as facetas articulares e de forma semelhante fixa-se um segundo pino de Steinmann do lado posto. Os pinos de Steinmann são fixados pelos fios nylon perpassando o orifício nas facetas articulares e na base do processo espinhoso deixando as extremidades longas para depois envolver os pinos longitudinalmente (Figura 1. B).

Foram estabilizadas a vertebras L5, L6, L7, e $\mathrm{S} 1$ e S2. Os pinos pré-colocados foram suturados com no mínimo 5 nós, e fixados longitudinalmente. A ferida cirúrgica foi lavada com soro fisiológico estéril aquecido. As extremidades dos pinos foram fechadas com a fáscia glútea. Os músculos epaxiais pela justaposição e sutura na linha média. 


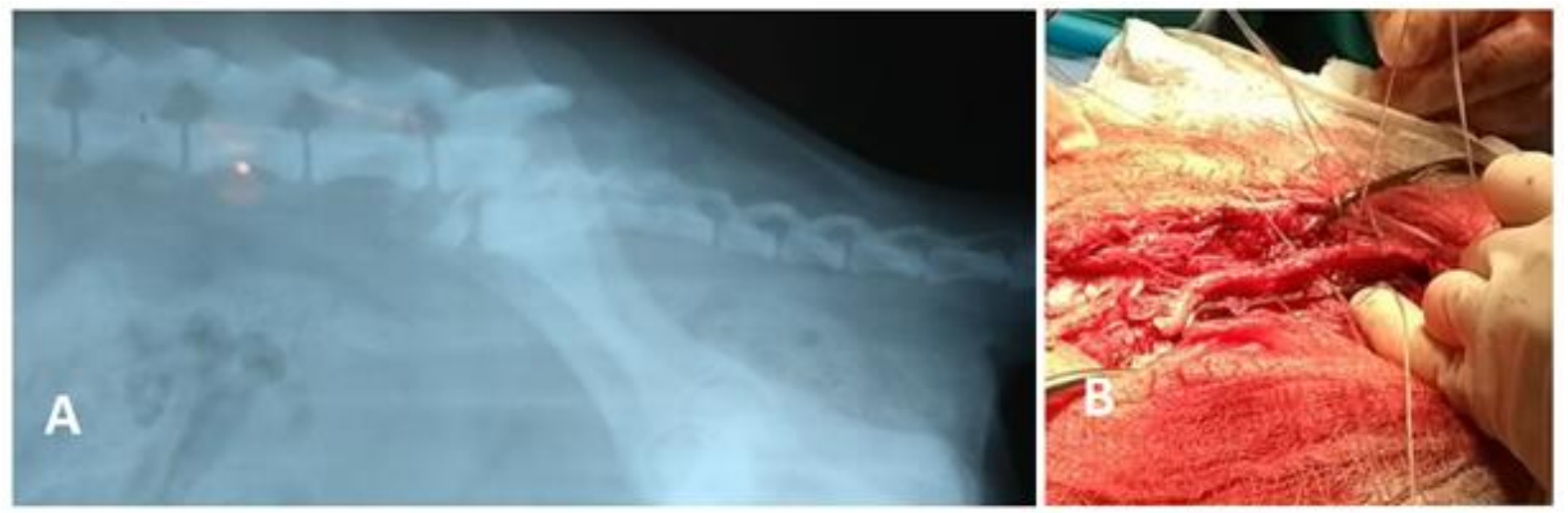

Figura 1. (A) Radiografia observa-se fratura entre as vertebras L7 e S1, presença de esquirola ósseas do corpo da vertebra (B) Observa-se os fios Nylon $(0,6 \mathrm{~mm})$ preso às facetas articulare.

\section{Discussão}

O paciente devido à fratura apresentava síndrome da cauda equina aguda. Semelhante ao que foi descrito por Chrisman (1985) e Inzana \& Wood (2008), o animal apresentava dor no dorso, com reflexo perianal ausente e diminuição da propriocepção e paresia dos membros pélvicos, mantinha o dorso flexionado, recusava a subir escadas e a sentar-se, apresentava reflexos ainda presentes de dor profunda e dor superficial, sem presença de atrofia muscular.

A dor nas fraturas lombosacrais é intensa e a correção cirúrgica causa melhor conforto na recuperação do animal, além de evitar a formação de déficit neurológica nos nervos ciático e pudendo (Weh \& Kraus, 2012).

A técnica de fixação interna produz estabilidade para resistir a encurvamento significante permitindo uma rápida recuperação. No entanto, justifica-se o uso da técnica de fixação segmentar modificada por pinos de Steinmann, por se tratar de uma técnica de baixo custo e fácil aplicação (Mcanulty et al., 1986).

$\mathrm{Na}$ recuperação pós cirúrgicos o paciente apresentou um comportamento ativo, sem presença de dor, se locomovendo bem, com defecação e micção dentro da normalidade, reflexos e propriocepção normal, apenas ainda relutante, ao subir escadas indica que ainda não houve uma recuperação completa do tônus muscular (Chrisman, 1985; Prata, 1998).

\section{Conclusão}

As fraturas lombo sacrais são comuns em cães em gatos, por esta razão que o estudo das técnicas de fixação vertebral interna e estabilização do canal vertebral tem grande importância na clínica da medicina veterinária. $\mathrm{O}$ que se pode verificar é que a técnica de fixação segmentar foi um sucesso na estabilização da coluna e na recuperação do déficit neurológicos já apresentados pelo paciente. A simplicidade e o baixo custo da técnica torna-se uma opção de grande relevância clínica para as fraturas lombo sacrais.

\section{Referências}

Curtis, W. D. 2014. Cirurgia da Cauda Equina. In: Fossum, T. W. Cirurgias de pequenos Animais (4a ed., p. 1529-1539). Elsevier, Rio de Janeiro, Rio de Janeiro, BR.

Chrisman, C. L. 1985. Paresia ou Paraplegia de um Membro. In: Neurologia dos pequenos animais (cap. 18, p. 371-386). Roca, São Paulo, São Paulo, BR.

Feitosa, M. M. 2014. Semiologia do Sistema Nervoso de Pequenos Animais In: Semiologia Veterinária: A arte do Diagnóstico ( $3^{\mathrm{a}} \mathrm{ed}$.). Roca, São Paulo, BR.

Inzana. K. D. \& Wood, B. C. 2008. Estenose lombo sacral em síndrome da cauda equina. In: Tilley, L.P. \& Smith Junior, F. W. K. Consulta veterinária em 5 minutos: espécie canina e felina (3a ed., p. 564-565). Manole, Barieri, São Paulo, BR.

Mcanulty, J. F., Lenehan, T. M. \& Maletz, L. M. 1986. Modified segmental spinal stabilization in repair of spinal fractures and luxations in dogs. Veterinary Surgery, 15,143-149.

Prata, R. G. Síndrome da cauda Equina. 1998. In: Slatter, D. Manual de cirurgia de pequenos animais (2a ed., p. 1314-1331). Manole, São Paulo, São Paulo, BR.

Sein III, H. B. 2008. Cirurgias da coluna Lombo Sacral. In: Sein. T. W. Cirurgias de Pequenos Animais (cap. 40, p. 1493-1513). Elsevier, Rio de Janeiro, Rio de Janeiro, BR. 
Weh, M. \& Kraus, K. H. 2012. Spinal fractures and luxations. In: Tobias, K. M. \& Johnston, S. A. Veterinary surgery small animals (p. 487503). Elsevier-Saunders, Philadelphia, US. Article History:
Received 3 March 2018

Accepted 13 April 2018

Available online 31 May 2018

License information: This is an open-access article distributed under the terms of the Creative Commons Attribution License 4.0, which permits unrestricted use, distribution, and reproduction in any medium, provided the original work is properly cited. 